\title{
Severity of Vitamin D Deficiency Predicts Mortality in Ischemic Stroke Patients
}

\author{
Jarosław Wajda $\left(\mathbb{D},{ }^{1}\right.$ Maciej Świat, ${ }^{2,3}$ Aleksander J. Owczarek $\mathbb{D},{ }^{4}$ Aniceta Brzozowska, ${ }^{5}$ \\ Magdalena Olszanecka-Glinianowicz, ${ }^{5}$ and Jerzy Chudek (iD ${ }^{6,7}$ \\ ${ }^{1}$ Dialysis Center in Rybnik, Regional Specialist Hospital No. 3 in Rybnik, Poland \\ ${ }^{2}$ Department of Neurology with Stroke Unit, Regional Specialist Hospital No. 3 in Rybnik, Poland \\ ${ }^{3}$ Jan Dlugosz University in Czestochowa, Poland \\ ${ }^{4}$ Department of Statistics, Department of Instrumental Analysis, Faculty of Pharmacy and Laboratory Medicine in Sosnowiec, \\ Medical University of Silesia, Katowice, Poland \\ ${ }^{5}$ Health Promotion and Obesity Management Unit, Department of Pathophysiology, Medical Faculty in Katowice, Medical University \\ of Silesia, Katowice, Poland \\ ${ }^{6}$ Pathophysiology Unit, Department of Pathophysiology, Medical Faculty in Katowice, Medical University of Silesia, Katowice, Poland \\ ${ }^{7}$ Department of Internal Medicine and Oncological Chemotherapy, Medical Faculty in Katowice, Medical University of Silesia, \\ Katowice, Poland
}

Correspondence should be addressed to Jarosław Wajda; jawaj@op.pl

Received 18 November 2018; Revised 17 February 2019; Accepted 3 March 2019; Published 2 May 2019

Academic Editor: Tomás Sobrino

Copyright ( 2019 Jarosław Wajda et al. This is an open access article distributed under the Creative Commons Attribution License, which permits unrestricted use, distribution, and reproduction in any medium, provided the original work is properly cited.

Background. Vitamin D (VD) deficiency is considered an independent risk factor for death due to cardiovascular events including ischemic stroke (IS). We assessed the hypothesis that decreased levels of 25 -hydroxyvitamin D (25-OH-D) are associated with increased risk of mortality in patients with IS. Methods. Serum 25-OH-D, intact parathyroid hormone (iPTH), and intact fibroblast growth factor 23 (iFGF23) levels were assessed in serum of 240 consecutive patients admitted within the 24 hours after the onset of IS. Mortality data was obtained from the local registry office. Results. Only three subjects (1.3\%) had an optimal 25-OH-D level (30-80 ng/mL), 25 (10.4\%) had a mildly reduced (insufficient) level, 61 (25.4\%) had moderate deficiency, and $151(62.9 \%)$ had a severe VD deficiency. 20\% subjects had secondary hyperparathyroidism. The serum 25-OH-D level was significantly lower than that in 480 matched subjects ( $9.9 \pm 7.1$ vs. $21.0 \pm 8.7 \mathrm{ng} / \mathrm{mL}$ ). Of all the patients, $79(32.9 \%)$ died during follow-up observation (44.9 months). The mortality rates (per year) were 4.81 and 1.89 in a group with and without severe VD deficiency, respectively (incidence rate ratio: 2.52; 95\% CI: 1.44-4.68). There was no effect of secondary hyperparathyroidism and iFGF23 levels on mortality rates. Age, $25-\mathrm{OH}-\mathrm{D}<10 \mathrm{ng} / \mathrm{mL}$, and functional status (modified Rankin scale) were significant factors increasing the risk of death in multivariable Cox proportional hazard regression test. Conclusions. Severe VD deficiency is an emerging, strong negative predictor for survival after IS, independent of age and functional status. VD supplementation in IS survivals may be considered due to high prevalence of its deficiency. However, it is uncertain whether it will improve their survival.

\section{Background}

Ischemic stroke occurs in all ages on all continents, often causing irreversible damage to the brain, and is the most common cause of disability in the people over 45 years old. Moreover, strokes are the third leading cause of death [1].
Despite major improvements in primary prevention and acute treatment over the last decades, stroke is still a devastating disease. At the beginning of the 21st century, the agestandardized incidence of stroke in Europe ranged from 95 to $290 / 100,000$ per year, with one-month case-fatality rates ranging from 13 to $35 \%$. Approximately 1.1 million 
European inhabitants suffered a stroke each year, and IS accounted for approximately $80 \%$ of cases [2].

The established risk factors for IS do not explain the occurrence of the disease in one-third of patients [3], justifying further exploration of potential risk factors. Recently, increased frequency of vitamin D (VD) deficiency was found in patients with cardiovascular diseases [4-6], including IS [7], and the association between VD deficiency and increased risk of death due to cardiovascular events was shown [8].

There are a few potential mechanisms that may explain the relationship between VD deficiency and the occurrence of IS. One of the most important is the development of secondary hyperparathyroidism [4-6] with disturbances related to the excess of PTH: insulin resistance, dysfunction of pancreatic beta cells predisposing to type 2 diabetes, hypertension, and dyslipidemia development [5]. Furthermore, activation of the renin-angiotensin system leading to increased blood pressure, stimulation of inflammation, and endothelial dysfunction cause progression of atherosclerosis [6]. In addition, VD may exert a neuroprotective effect by inhibiting inducible nitric oxide synthase (iNOS) that is increased during ischemic events, as well as antioxidant and anti-inflammatory processes [9].

In the present study, we assessed the hypothesis that decreased levels of 25-hydroxyvitamin D (25-OH-D) and calcium-phosphate disturbances are associated with increased risk of mortality in patients with IS.

\section{Patients and Methods}

It was a retrospective cohort study utilizing frozen serum samples that remained after routine laboratory tests in the hospital laboratory, obtained in consecutive patients with acute IS, admitted to the Stroke Unit of the Department of Neurology, Regional Specialist Hospital No. 3 in Rybnik (from January 2013 to August 2015) within 24 hours after the onset of symptoms. The utilization of serum samples and retrieval of data from medical reports without obtaining an informed consent were approved by the Bioethics Committee of the Medical University of Silesia.

Data concerning clinical status, comorbidities, medication, previous ischemic cerebrovascular episodes, cardiovascular risk factors, and laboratory parameters (C-reactive protein, glucose, creatinine, lipid profile, and urinalysis) were obtained from hospital medical files (routine work-up). Follow-up data for subsequent ischemic strokes were obtained from hospital medical records, which were checked quarterly, during and after the observation period for the entire study group, as well as from medical records of general practitioners, and mortality from the Registry Office in Rybnik (last actualization for 31 December 2016).

The diagnosis of acute IS was based on neurological examination and was supported with computed tomography (CT), after exclusion of other causes of acute neurological deficit (including hemorrhagic stroke), and in accordance with current guidelines [10]. Imaging studies were positive for ischemic changes typical for the acute phase of IS and correlated with clinical symptoms. The course of IS was evaluated on the basis of the severity of neurological deficit in the NIHSS (National Institute of Health Stroke Scale) and functional status at discharge using the modified Rankin scale (mRS). Additional studies determined the probable mechanism of IS, according to the TOAST (Trial of Org 10172 in Acute Stroke Treatment) classification [11].

Patients with hemorrhagic stroke, history of cancer, apparent inflammation, and impairment in activities of daily living before the stroke (ADL less than 5 points in the Katz Index) were excluded from the analysis.

2.1. Control Group. The group consisted of 480 subjects drawn from a population-based community dwelling cohort of older subjects (PolSenior study [12]) without a history of cancer, matched according to sex, age ( $73 \pm 6$ years), occurrence of diabetes (34.8\%), and hypertension $(81.4 \%)$.

2.2. Measurements. Additional assessments were performed in stored frozen samples in the laboratory of the Department of Pathophysiology. Serum 25-OH-D (limit of quantification $3 \mathrm{ng} / \mathrm{mL}$ ) and intact parathyroid hormone (iPTH) levels were assessed by an electrochemiluminescence method on a Cobas E411 analyzer (Roche Diagnostics $\mathrm{GmbH}$, Mannheim, Germany) with inter-assay coefficients of variability below 7.8 and $6.5 \%$, respectively. We measured serum intact fibroblast growth factor 23 (iFGF23) (Immutopics, San Clemente, CA, U.S.) and high-sensitivity (limit of quantification $(0.09 \mathrm{mg} / \mathrm{L})$ C-reactive protein (Immundiagnostik AG, Bensheim, Germany) concentrations by ELISA. The mean intra- and interassay coefficients were $4.4 \%$ and $6.1 \%$ for iFGF23 and $<6 \%$ and $<11.6 \%$ for hsCRP, respectively.

Serum phosphorus and calcium were assessed by an automated system (Cobas 111, Roche Diagnostics $\mathrm{GmbH}$, Mannheim, Germany) with interassay coefficients of variability below 2.3 and $1.3 \%$, respectively.

2.3. Data Analysis. VD status was categorized by commonly used cutoffs and definitions of serum 25-OH-D: values < $10 \mathrm{ng} / \mathrm{mL}$ were categorized as a severe deficiency, between 10 and $19.9 \mathrm{ng} / \mathrm{mL}$ as a deficiency, between 20 and $29.9 \mathrm{ng} / \mathrm{mL}$ as insufficiency, and those above $30 \mathrm{ng} / \mathrm{mL}$ as sufficient 25-OH-D concentrations [13].

Patients with iPTH above $65 \mathrm{pg} / \mathrm{mL}$ and normal or low total calcium were diagnosed with secondary hyperparathyroidism.

Estimated glomerular filtration rate (eGFR) was estimated according to the short MDRD formula (Modification of Diet in Renal Disease) [14]. Patients with $\mathrm{eGFR}<60 \mathrm{~mL} / \mathrm{min} / 1.73 \mathrm{~m}^{2}$ or albuminuria in routine urinalysis were considered as having kidney function impairment.

2.4. Study End Points. The primary outcome was death, and the secondary outcome was composite end point (CEP) stated as death or a recurrent IS.

2.5. Statistical Analysis. Statistical analysis was performed using STATISTICA 10.0 PL (StatSoft, Cracow, Poland), StataSE 13.0 (StataCorp LP, TX, U.S.), and R software. Statistical significance was set at a $p$ value below 0.05 . All tests were 
two-tailed and data imputation was not performed. Nominal and ordinal data were expressed as percentages, whilst interval data was expressed as mean value \pm standard deviation in the case of a normal distribution or as median with lower and upper quartiles in the case of data with skewed or nonnormal distribution. Distribution of variables was evaluated by the Anderson-Darling test. Homogeneity of variances was assessed by the Levene test.

In order to show survival rate and cumulative hazard estimates according to the follow-up time, Kaplan-Meier curves and Nelson-Aalen estimates were used with a logrank test to compare survival distribution between two samples. Risk factors of death, as well as CEP, were analyzed with univariable and multivariable stepwise backward Cox proportional hazard regression. All statistically significant factors in univariable analysis were included into a multivariable stepwise backward model ( $p>0.05$ for removal). In the case of age, we used median as a cutoff point.

Schoenfeld residuals were used to test the proportional hazard (PH) assumption. The concordance probability was calculated with Gönen and Heller's $K$ concordance coefficient. The functional status was included into the analysis, based on the modified Rankin scale (mRS), at admission equal or higher than 4 pts, while at discharge equal to 5 pts, according to statistical significance of the hazard ratio.

\section{Results}

3.1. Study Group Characteristics. Among 240 study patients, $17.5 \%(N=42)$ had a previous history of IS, $1.7 \%(N=4)$ of transient ischemic attack, and $1.7 \%(N=4)$ of intracerebral bleeding. Cardiovascular diseases (particularly hypertension, $84.6 \%)$ and diabetes (35.0\%) were highly prevalent in the study group (Table 1 ).

On admission, $27.0 \%$ were without or with minor (NIHSS $\leq 4$ pts), $54.2 \%$ with moderate (5-15 pts), and $18.8 \%$ with moderate to severe or severe IS symptoms ( $\geq 16$ pts). Large vessel occlusion was the most prevalent cause of IS $(N=106 ; 44.2 \%)$ followed by lacunar $(N=56$; $23.3 \%)$, embolic stroke $(N=55 ; 22.9 \%)$, other classified $(N=8 ; 2.3 \%)$, and unclassified $(N=15 ; 6.3 \%)$.

3.2. VD Levels. Upon hospital admission, only three subjects (1.3\%) had a $25-\mathrm{OH}-\mathrm{D}$ level within the optimal range, 25 (10.4\%) had mildly reduced (insufficient) levels, 61 (25.4\%) had moderate deficiency, and 151 (62.9\%) had severe deficiency. One patient was supplemented with VD and 2 were treated with bisphosphonates. There was no patient taking calcium-containing supplements.

In the study group, the serum 25-OH-D level was significantly lower than that in the control group $(9.9 \pm 7.1$ vs. $21.0 \pm 8.7 \mathrm{ng} / \mathrm{mL} ; p<0.001)$. In the control group, 163 $(34.0 \%)$ had mildly reduced (insufficient) levels, 217 (45.2\%) moderate deficiency, and only 29 (6.0\%) severe deficiency.

There was a greater prevalence of secondary hyperparathyroidism in IS patients $(N=48 ; 20 \%)$ than in the control subjects $(N=65 ; 13.7 \% ; p<0.05)$.
Similar 25-OH-D and iPTH levels were observed in patients regardless of the type of IS (Table 1).

3.3. Hospitalization Period. Thrombolysis was performed in 36 patients. During hospitalization, 24 patients (10\%) died: $8(44.4 \%)$ with NIHSS > 20, 6 (22.2\%) with NIHSS 16-20, $8(6.1 \%)$ with NIHSS 5-15, and 2 (3.2\%) with NIHSS 1-4. Similar percentages of fatal outcomes were observed in patients after thrombolysis and treated conservatively ( $8.3 \%$ vs. $10.3 \%$, respectively).

At discharge, 85 patients (39.4\% of survivals) had no significant disability (0-1 in mRS), 32 (14.8\%) slight disability ( 2 in $\mathrm{mRS}), 36$ (16.7\%) moderate disability ( 3 in $\mathrm{mRS}$ ), $31(14.3 \%)$ moderately severe disability (4 in $\mathrm{mRS}$ ), and $32(14.8 \%)$ sever disability (5 in mRS) (Table 2).

Patients with beneficial outcome (mRS 0-1) had higher 25-OH-D levels than those with fatal outcome $\left(9.0\left(Q_{1}-Q_{3}\right.\right.$ : 4.9-14.3) vs. 6.3 (3.4-9.8) $\mathrm{ng} / \mathrm{mL}, p<0.05$, respectively). The difference in corresponding iPTH values was not significant (37.1 $\left(Q_{1}-Q_{3}: 22.3-52.0\right)$ vs. $\left.47.4(35.2-60.7), p=0.2\right)$.

3.4. VD and Survival. The period of the follow-up was up to 48 months (mean 25 months). The primary outcome, death, was noted in 79 (32.9\%) subjects during the period of followup. Median survival time and extended mean survival time were 44.9 and 64.5 months, respectively. The mortality rates (per year) were 4.81 and 1.89 in groups with and without severe VD deficiency, respectively, with an incident rate ratio equal to 2.52; (95\% CI: 1.44-4.68) (Figure 1). Furthermore, we estimated that $60.4 \%$ (95\% CI: $30.6 \%-76.6 \%)$ of deaths could be assigned to VD deficiency (population attributable risk (PAR)). Therefore, we estimated it would be necessary to treat 5 (95\% CI: $2.9-8.0)$ subjects with severe VD deficiency to prevent one death (number needed to treat (NNT)).

The log-rank test for crude as well as for age-adjusted analysis proved differences between survival curves $\left(\chi^{2}=\right.$ $10.9, p<0.01$ and $\chi^{2}=9.5, p<0.01$, respectively). The difference in survival probability between the groups began after 15.8 months of follow-up. Nelson-Aalen cumulative hazard estimates confirmed a higher hazard rate of death in the group with severe VD deficiency (Figure 2).

According to univariable Cox proportional hazard regression, age over 72 years, female gender, diabetes mellitus and atrial fibrillation occurrence, serum iPTH $\geq 65 \mathrm{pg} / \mathrm{mL}$, $25-\mathrm{OH}-\mathrm{D}<10 \mathrm{ng} / \mathrm{mL}$, and $\mathrm{eGFR}<60 \mathrm{~mL} / \mathrm{min} / 1.73 \mathrm{~m}^{2}$ as well as a patient's $\mathrm{mRS} \geq 4$ points at admission were risk factors for death. After adjustment for age, female gender, atrial fibrillation occurrence, serum iPTH $\geq 65 \mathrm{pg} / \mathrm{mL}$, and eGFR $<60 \mathrm{~mL} / \mathrm{min} / 1.73 \mathrm{~m}^{2}$ lost their statistical significance. Patient's mRS at discharge $=5$ was the only factor that did not fulfil proportional hazard assumption (also after age adjustment) and was incorporated into the analysis as a time-dependent factor that proved to increase the risk of death (Table 3).

In multivariable stepwise backward Cox proportional hazard regression, age over 72 years, serum $25-\mathrm{OH}-\mathrm{D}<$ $10 \mathrm{ng} / \mathrm{mL}$, and $\mathrm{mRS}$ at discharge proved to be statistically significant factors increasing the risk of death (Table 4). Based on Gönen and Heller's $K$ concordance coefficient, we 
TABLE 1: Patients' characteristics and comparison of groups with three main stroke types.

\begin{tabular}{|c|c|c|c|c|}
\hline & $\begin{array}{l}\text { All subjects } \\
(N=240)\end{array}$ & $\begin{array}{l}\text { Large vessel stroke } \\
\qquad(N=106)\end{array}$ & $\begin{array}{l}\text { Lacunar } \\
(N=56)\end{array}$ & $\begin{array}{l}\text { Embolic } \\
(N=55)\end{array}$ \\
\hline Age (years) & $72 \pm 11$ & $70 \pm 12$ & $70 \pm 9$ & $78 \pm 10^{* * *, \wedge \wedge \wedge}$ \\
\hline Gender (men/women) & $109 / 131$ & $49 / 57$ & $33 / 23$ & $17 / 38$ \\
\hline History of previous stroke $(n / \%)$ & $42 / 17.5$ & $15 / 14.1$ & $9 / 16.1$ & $15 / 27.3$ \\
\hline \multicolumn{5}{|l|}{ Concomitant diseases } \\
\hline Hypertension $(n / \%)$ & $203 / 84.6$ & $88 / 83.0$ & $48 / 85.7$ & $50 / 90.9$ \\
\hline Coronary artery disease $(n / \%)$ & $69 / 28.8$ & $32 / 30.2$ & $9 / 16.1$ & $22 / 40.0^{* *}$ \\
\hline Valvular diseases $(n / \%)$ & $24 / 10.0$ & $10 / 9.4$ & $5 / 8.9$ & $9 / 16.4$ \\
\hline Atrial fibrillation $(\mathrm{AF})(n / \%)$ & $70 / 29.2$ & $14 / 13.2$ & $5 / 8.9$ & $47 / 85.4^{* * *, \wedge \wedge \wedge}$ \\
\hline Antithrombotic agents in AF patients $(n / \%)$ & $23 / 32.9$ & $4 / 28.6$ & 0 & $19 / 40.4$ \\
\hline Symptomatic atherosclerosis $(n / \%)$ & $101 / 42.1$ & $52 / 49.1$ & $19 / 33.9$ & $23 / 41.8$ \\
\hline Obesity $(n / \%)$ & $44 / 18.3$ & $19 / 17.9$ & $11 / 19.6$ & $12 / 21.8$ \\
\hline Diabetes $(n / \%)$ & $84 / 35.0$ & $37 / 34.9$ & $19 / 33.9$ & $23 / 41.8$ \\
\hline Hypercholesterolemia $(n / \%)$ & $107 / 44.6$ & $49 / 46.2$ & $29 / 51.8$ & $23 / 41.8$ \\
\hline Statin therapy $(n / \%)$ & $92 / 38.3$ & $41 / 38.7$ & $18 / 32.1$ & $25 / 45.4$ \\
\hline Active smokers $(n / \%)$ & $45 / 18.7$ & $23 / 21.7$ & $17 / 30.4$ & $5 / 9.1^{* *}$ \\
\hline $\begin{array}{l}\text { The National Institutes of Health Stroke Scale index (NIHSS-I) } \\
\text { at admission }\end{array}$ & $9 \pm 6$ & $9 \pm 6$ & $6 \pm 5$ & $12 \pm 7^{* * *}$ \\
\hline \multicolumn{5}{|l|}{ Modified Rankin scale (mRS) at discharge $(n / \%)$} \\
\hline $0-1$ (no significant disability) & $85 / 26.7$ & $40 / 37.7$ & $26 / 46.4$ & $12 / 21.8$ \\
\hline 2 (mild disability) & $32 / 13.3$ & $15 / 14.2$ & $9 / 16.1$ & $7 / 12.7$ \\
\hline 3 (moderate disability) & $36 / 15.0$ & $11 / 10.4$ & $11 / 19.6$ & $9 / 16.4$ \\
\hline 4-5 (moderately severe and severe disability) & $63 / 26.2$ & $31 / 29.2$ & $7 / 12.5$ & $20 / 36.4$ \\
\hline 6 (death) & $24 / 10.0$ & $9 / 8.5$ & $3 / 5.4$ & $7 / 12.7$ \\
\hline One-year survival $(n / \%)$ & $167 / 75.0$ & $79 / 74.5$ & $49 / 87.5$ & $39 / 70.9$ \\
\hline Two-year survival $(n / \%)$ & $96 / 70.0$ & $52 / 68.5$ & $17 / 87.5$ & $27 / 63.0$ \\
\hline Total cholesterol (mmol/L) & $5.1 \pm 1.5$ & $5.1 \pm 1.4$ & $5.2 \pm 1.9$ & $4.8 \pm 1.3$ \\
\hline LDL-cholesterol (mmol/L) & $3.2 \pm 1.3$ & $3.3 \pm 1.1$ & $3.8 \pm 1.4$ & $2.4 \pm 0.9^{* * *, \wedge \wedge \wedge}$ \\
\hline HDL-cholesterol (mmol/L) & $1.4 \pm 0.4$ & $1.4 \pm 0.4$ & $1.5 \pm 0.3$ & $1.4 \pm 0.5$ \\
\hline Triglycerides $(\mathrm{mmol} / \mathrm{L})$ & $1.7 \pm 1.0$ & $1.7 \pm 0.8$ & $1.8 \pm 1.2$ & $1.3 \pm 0.8$ \\
\hline Creatinine $(\mu \mathrm{mol} / \mathrm{L})$ & $89 \pm 51$ & $\mathrm{~N} / \mathrm{A}$ & $\mathrm{N} / \mathrm{A}$ & N/A \\
\hline Estimated glomerular filtration rate (eGFR) $\left(\mathrm{mL} / \mathrm{min} / 1.73 \mathrm{~m}^{2}\right)$ & $70 \pm 25$ & $73 \pm 26$ & $72 \pm 23$ & $61 \pm 22^{\wedge \wedge}$ \\
\hline $\mathrm{eGFR}<60 \mathrm{~mL} / \mathrm{min} / 1.73 \mathrm{~m}^{2}(n / \%)$ & $86 / 35.8$ & $31 / 29.2$ & $20 / 35.7$ & $29 / 52.7^{\wedge \wedge}$ \\
\hline C-reactive protein (CRP) (mg/L) & $4.2(1.8-9.3)$ & $5.1(2.0-9.5)$ & $3.3(1.4-8.4)$ & $3.5(1.7-8.1)$ \\
\hline Calcium $(\mathrm{mg} / \mathrm{dL})$ & $2.32 \pm 0.20$ & $2.31 \pm 0.19$ & $2.33 \pm 0.20$ & $2.24 \pm 0.20$ \\
\hline Phosphorus (mg/dL) & $1.11(0.96-1.33)$ & $1.09(0.95-1.32)$ & $1.22(0.96-1.48)$ & $1.11(0.97-1.28)$ \\
\hline Intact parathyroid hormone (iPTH) (pg/mL) & $40.7(26.7-56.4)$ & $39.0(24.4-56.5)$ & $39.5(21.7-63.0)$ & $42.2(31.1-61.2)$ \\
\hline $\mathrm{iPTH}>65 \mathrm{pg} / \mathrm{mL}(n / \%)$ & $48 / 20.0$ & $19 / 17.9$ & $14 / 25.0$ & $12 / 21.8$ \\
\hline Intact fibroblast growth factor 23 (iFGF23) (pg/mL) & $50.0(17.6-85.4)$ & $52.1(21.6-92.2)$ & $66.7(23.9-84.4)$ & $42.4(18.5-71.7)$ \\
\hline iFGF23 > $50 \mathrm{pg} / \mathrm{mL}(n / \%)$ & $120 / 50.0$ & $54 / 50.9$ & $34 / 60.7$ & $25 / 45.5$ \\
\hline $25-\mathrm{OH}-\mathrm{D}(\mathrm{ng} / \mathrm{mL})$ & $9.9 \pm 7.1$ & $9.7 \pm 6.9$ & $11.1 \pm 8.2$ & $9.4 \pm 6.6$ \\
\hline $25-\mathrm{OH}-\mathrm{D}<10 \mathrm{ng} / \mathrm{mL}(n / \%)$ & $151 / 62.9$ & $66 / 62.3$ & $33 / 58.9$ & $38 / 69.1$ \\
\hline
\end{tabular}

${ }^{* *} p<0.01 ;{ }^{* * *} p<0.001$ vs. lacunar stroke. ${ }^{\wedge \wedge} p<0.01 ;{ }^{\wedge \wedge} p<0.001$ vs. large vessel stroke.

may assume that we can correctly identify survival times for pairs of patients $72.2 \%$ in crude and $78.3 \%$ in an age-adjusted analysis of the time on the basis of the mentioned factors.
3.5. VD and Composite End Point (Death or Recurrent Stroke). Of all patients, 85 (35.4\%) had a composite end point (CEP). Lower quartile and extended mean survival time were of $4.6 / 46.5$ and $29.5 / 137.5$ months, respectively. The 
TABLE 2: Comparison of subgroups distinguished according to the modified Rankin scale at discharge.

\begin{tabular}{|c|c|c|c|c|}
\hline & \multicolumn{4}{|c|}{ Modified Rankin scale at discharge } \\
\hline & $\begin{array}{c}0-1 \\
\text { (no significant } \\
\text { disability) } \\
(N=85)\end{array}$ & $\begin{array}{c}2-3 \\
\text { (mild/moderate } \\
\text { disability) } \\
(N=68)\end{array}$ & $\begin{array}{c}4-5 \\
\text { (moderately severe } \\
\text { and severe disability) } \\
(N=63)\end{array}$ & $\begin{array}{c}6 \\
(\text { death }) \\
(N=24)\end{array}$ \\
\hline Age (years) & $67 \pm 11$ & $71 \pm 10$ & $76 \pm 11^{\wedge \wedge \wedge}$ & $79 \pm 11^{\wedge \wedge \wedge}$ \\
\hline Gender (men/women) & $48 / 37$ & $33 / 35$ & $18 / 45$ & $10 / 14$ \\
\hline History of previous stroke $(n / \%)$ & $14(16.5)$ & $14(20.6)$ & $10(15.9)$ & $4(16.7)$ \\
\hline \multicolumn{5}{|l|}{ Concomitant diseases } \\
\hline Hypertension $(n / \%)$ & $71(83.5)$ & $60(88.2)$ & $54(85.7)$ & $18(75.0)$ \\
\hline Coronary artery disease $(n / \%)$ & $26(30.6)$ & $15(22.1)$ & $20(31.8)$ & $8(33.3)$ \\
\hline Valvular diseases $(n / \%)$ & $7(8.2)$ & $7(10.3)$ & $9(14.3)$ & $1(4.2)$ \\
\hline Atrial fibrillation $(n / \%)$ & $17(20.0)$ & $15(22.1)$ & $27(42.9)^{\wedge \wedge}$ & $11(45.8)^{\wedge}$ \\
\hline Obesity $(n / \%)$ & $18(21.2)$ & $8(12.8)$ & $16(25.4)$ & $2(8.3)$ \\
\hline Diabetes $(n / \%)$ & $25(29.4)$ & $22(32.3)$ & $26(41.3)$ & $11(45.8)$ \\
\hline Hypercholesterolemia $(n / \%)$ & $37(43.5)$ & $35(51.5)$ & $29(46.0)$ & $6(25.0)$ \\
\hline Statin therapy $(n / \%)$ & $36(42.3)$ & $26(38.2)$ & $22(34.9)$ & $8(33.3)$ \\
\hline Active smokers $(n / \%)$ & $20(23.5)$ & $13(19.1)$ & $11(17.5)$ & $1(4.2)$ \\
\hline $\begin{array}{l}\text { Estimated glomerular filtration rate (eGFR) } \\
\left(\mathrm{mL} / \mathrm{min} / 1.73 \mathrm{~m}^{2}\right)\end{array}$ & $72.9 \pm 23.4$ & $74.4 \pm 29.2$ & $64.1 \pm 21.3$ & $67.1 \pm 26.0$ \\
\hline $\mathrm{eGFR}<60 \mathrm{~mL} / \mathrm{min} / 1.73 \mathrm{~m}^{2}(n / \%)$ & $20(23.5)$ & $22(32.3)$ & $32(50.8)^{\wedge \wedge \wedge}$ & $12(50.0)^{\wedge}$ \\
\hline C-reactive protein (CRP) (mg/L) & $3.8(1.3-9.3)$ & $4.3(2.6-9.1)$ & $4.2(1.7-10.6)$ & $4.3(8.5-8.5)$ \\
\hline Calcium $(\mathrm{mg} / \mathrm{dL})$ & $2.35 \pm 0.22$ & $2.31 \pm 0.21$ & $2.31 \pm 0.18$ & $2.28 \pm 0.17$ \\
\hline Phosphorus (mg/dL) & $1.13(0.98-1.31)$ & $1.16(0.94-1.43)$ & $1.11(0.96-1.32)$ & $1.05(0.98-1.24)$ \\
\hline Intact parathyroid hormone (iPTH) (pg/mL) & $36.5(21.7-49.8)$ & $37.2(24.9-57.1)$ & $46.9(31.1-66.9)^{\wedge}$ & $47.4(35.2-60.7)$ \\
\hline $\mathrm{iPTH}>65 \mathrm{pg} / \mathrm{mL}(n / \%)$ & $10(11.8)$ & $15(22.1)$ & $17(27.0)$ & $6(25.0)$ \\
\hline Intact fibroblast growth factor 23 (iFGF23) (pg/mL) & $42.3(10.6-77.2)$ & $58.7(17.8-92.4)$ & $51.5(25.3-92.2)$ & $48.2(19.9-102.1)$ \\
\hline iFGF23 > $50 \mathrm{pg} / \mathrm{mL}(n / \%)$ & $37(43.5)$ & $39(57.4)$ & $33(52.4)$ & $11(45.8)$ \\
\hline $25-\mathrm{OH}-\mathrm{D}(\mathrm{ng} / \mathrm{mL})$ & $9.6(4.8-14.7)$ & $8.4(4.9-13.4)$ & $7.2(3.0-12.1)$ & $6.3(3.4-9.8)$ \\
\hline $25-\mathrm{OH}-\mathrm{D}<10 \mathrm{ng} / \mathrm{mL}(n / \%)$ & $48(56.5)$ & $43(63.2)$ & $42(66.7)$ & $18(75.0)^{\wedge}$ \\
\hline One-year survival $N(\%)$ & $80(94.1)$ & $58(85.3)$ & $45(71.4)^{\wedge \wedge \wedge}$ & - \\
\hline Two-year survival $N(\%)$ & $60(91.7)$ & $40(82.1)$ & $27(57.8)^{\wedge \wedge \wedge}$ & - \\
\hline
\end{tabular}

${ }^{\wedge} p<0.05 ;{ }^{\wedge \wedge} p<0.01 ;{ }^{\wedge \wedge} p<0.001$ vs. minor stroke $(0-1)$.

incidence rates of CEP per year were 4.34 and 1.77 in a group with and without severe VD deficiency, respectively, with an incidence rate ratio equal to 2.42; (95\% CI: $1.42-4.34$ ) (Figure 1). Moreover, we estimated that 58.8\% (95\% CI: $29.7 \%-76.9 \%$ ) of CEPs were due to severe VD deficiency (PAR). Therefore, we estimate it would be necessary to treat 5 (95\% CI: 2.8-7.9) persons with severe VD deficiency in order to prevent one CEP (NNT).

The log-rank test for crude as well as for age-adjusted analysis proved differences between survival curves $\left(\chi^{2}=\right.$ 11.3, $p<0.001$ and $\chi^{2}=9.9, p<0.01$, respectively). The difference in survival probability for CEP between the groups began after 15.8 months of follow-up. Nelson-Aalen cumulative hazard estimates confirmed a higher hazard rate of CEP in the group with severe VD deficiency.

Table 5 shows results of crude as well as age-adjusted univariable Cox proportional hazard regression analysis. Independent risk factors for CEP were age over 72 years, diabetes, and coronary artery disease occurrence, severe
VD deficiency, eGFR $>60 \mathrm{~mL} / \mathrm{min} / 1.73 \mathrm{~m}^{2}$, and $\mathrm{mRS}$ at discharge $\geq 4$ points. Furthermore, an increased level of cerebrovascular incidence severity and an increased NIHSS were factors that increased the risk of CEP. With only age adjustment, serum $25-\mathrm{OH}-\mathrm{D}<10 \mathrm{ng} / \mathrm{mL}$, mRS, and cerebrovascular incidence severity proved to be statistically significant. A patient's mRS at discharge $=5$ was the only factor that did not fulfil the proportional hazard assumption after age adjustment and was incorporated into the analysis as a time-dependent factor that proved to increase the risk of CEP occurrence.

In multivariable stepwise backward Cox proportional hazard regression, age over 72 years, severe VD deficiency, and $\mathrm{mRS}$ proved to be statistically significant factors increasing the risk of CEP occurrence (Table 4.) Based on Gönen and Heller's $K$ concordance coefficient, we may assume that we can correctly order survival times for pairs of patients $70.2 \%$ in crude and $75.9 \%$ in an age-adjusted analysis of the time on the basis of previously mentioned factors. 
Kaplan-Meier survival for composite end point

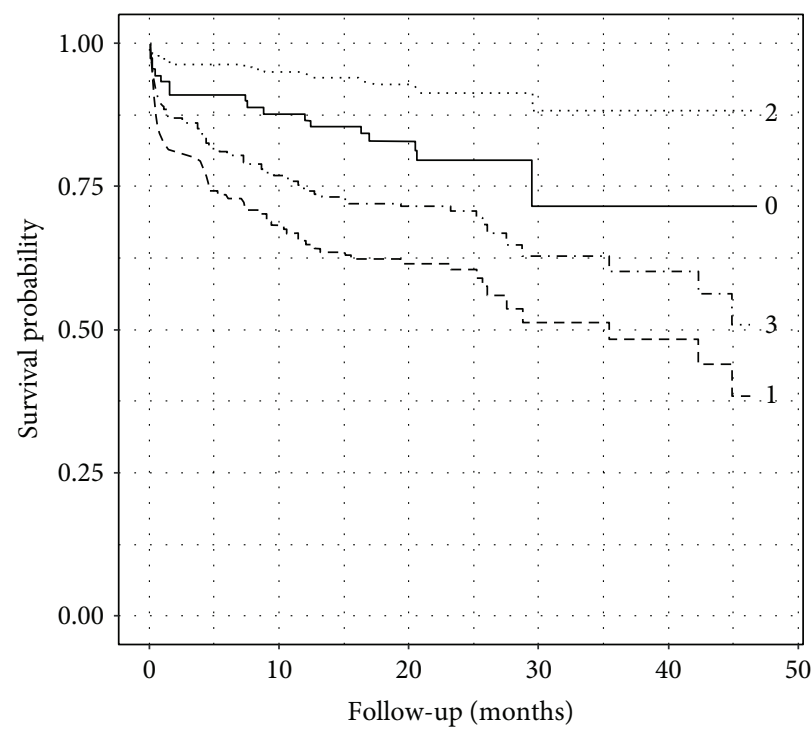

Group

- 0:25-OH- $\mathrm{D}_{3}>10(\mathrm{ng} / \mathrm{mL})$ crude

- _ - 1: 25-OH-D $<10(\mathrm{ng} / \mathrm{mL})$ crude

… 2: $25-\mathrm{OH}-\mathrm{D}_{3}>10(\mathrm{ng} / \mathrm{mL})$ age adjusted

3: $25-\mathrm{OH}-\mathrm{D}_{3}<10(\mathrm{ng} / \mathrm{mL})$ age adjusted

(a)

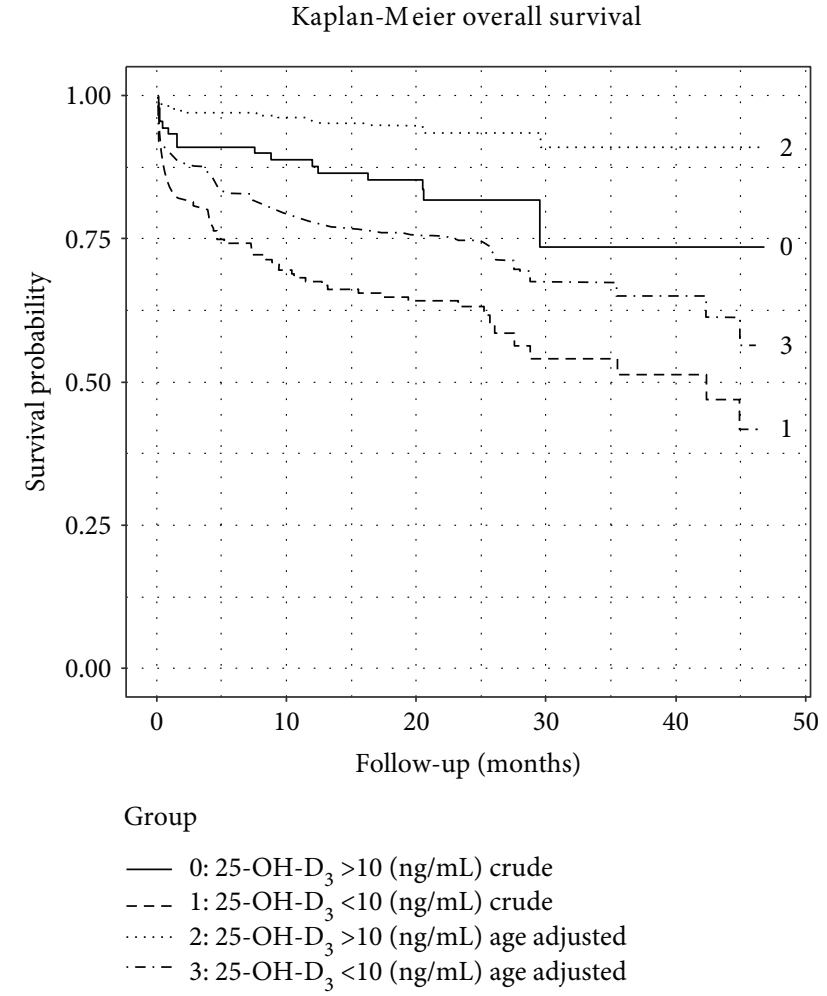

(b)

Figure 1: Kaplan-Meier crude and age-adjusted overall survival (a) and probability for composite end point (b) in groups with a 25-OH-D level lower and higher than $10(\mathrm{ng} / \mathrm{mL})$.

Nelson-Aalen cumulative hazard estimates

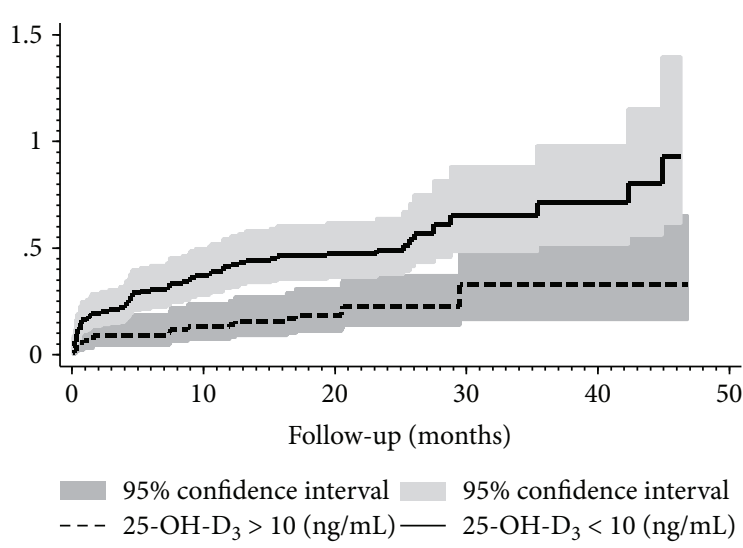

(a)
Nelson-Aalen cumulative hazard estimates

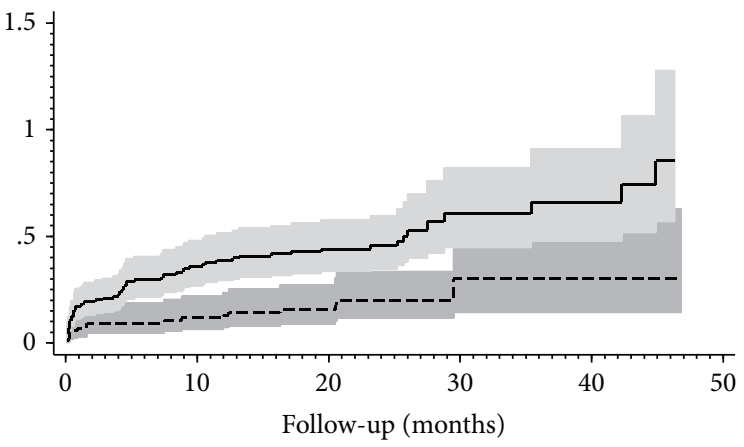

95\% confidence interval 95\% confidence interval $--25-\mathrm{OH}-\mathrm{D}_{3}>10(\mathrm{ng} / \mathrm{mL})-25-\mathrm{OH}-\mathrm{D}_{3}<10(\mathrm{ng} / \mathrm{mL})$

(b)

Figure 2: Nelson-Aalen cumulative hazard estimates of death (a) and composite end point (b) in groups with a 25-OH-D level lower and higher than $10(\mathrm{ng} / \mathrm{mL})$.

\section{Discussion}

In this study we show that almost all patients with IS, regardless of age and gender, had VD deficiency or insufficiency and that severe VD deficiency $(<10 \mathrm{ng} / \mathrm{mL})$, which is ten times more frequent than in community dwelling older adult matched according to sex, age, diabetes, and hypertension, independently of age and patient condition at discharge from the hospital assessed in mRS, is associated with increased mortality and therefore shorter after IS survival. It should be stressed that lower serum 25-OH-D in patients with unfavourable prognosis were not explained by preexisting cachexia and disability as well as inflammatory or neoplastic diseases, as these patients were excluded from the analysis.

Our results extend the findings of Sun et al. [15], who showed in a prospective observation an elevated (49\%) risk 
TABLE 3: Results of crude and age-adjusted univariable Cox proportional hazard regression for death.

\begin{tabular}{|c|c|c|c|c|c|c|c|c|c|c|}
\hline \multirow{2}{*}{ Parameter } & \multicolumn{5}{|c|}{ Cox proportional hazard—crude analysis } & \multicolumn{5}{|c|}{ Cox proportional hazard—age adjusted } \\
\hline & HR & $95 \%$ CI & $p$ & $\chi^{2}$ & $p$ & HR & $95 \%$ CI & $p$ & $\chi^{2}$ & $p$ \\
\hline Age $>72$ years & 2.74 & $1.69-4.43$ & $<0.001$ & 1.73 & 0.19 & - & - & - & - & - \\
\hline Female vs. male & 1.67 & $1.05-2.65$ & $<0.05$ & 0.33 & 0.57 & 1.27 & $0.78-2.07$ & 0.34 & 0.09 & 0.76 \\
\hline Diabetes & 1.79 & $1.15-2.78$ & $<0.05$ & 1.68 & 0.19 & 1.57 & $1.01-2.45$ & $<0.05$ & 1.11 & 0.29 \\
\hline Hypertension & 0.87 & $0.48-1.58$ & 0.65 & 1.65 & 0.20 & 0.71 & $0.38-1.29$ & 0.26 & 1.40 & 0.24 \\
\hline Atrial fibrillation & 1.68 & $1.07-2.64$ & $<0.05$ & 0.15 & 0.70 & 1.22 & $0.76-1.96$ & 0.40 & 0.34 & 0.55 \\
\hline Ischemic heart disease & 1.52 & $0.96-2.41$ & 0.08 & 0.02 & 0.90 & 1.32 & $0.83-2.11$ & 0.24 & 0.01 & 0.92 \\
\hline Valvular diseases & 1.67 & $0.88-3.17$ & 0.12 & 0.29 & 0.59 & 1.42 & $0.75-2.71$ & 0.28 & 0.44 & 0.51 \\
\hline Previous stroke & 1.14 & $0.67-1.92$ & 0.63 & 1.59 & 0.21 & 0.99 & $0.59-1.69$ & 0.99 & 1.57 & 0.21 \\
\hline Hypercholesterolemia & 0.72 & $0.43-1.19$ & 0.20 & 0.20 & 0.65 & 0.76 & $0.46-1.26$ & 0.29 & 0.21 & 0.64 \\
\hline $\mathrm{iPTH} \geq 65 \mathrm{pg} / \mathrm{mL}$ & 1.64 & $1.01-2.69$ & $<0.05$ & 0.18 & 0.67 & 1.41 & $0.86-2.32$ & 0.17 & 0.50 & 0.48 \\
\hline iFGF23 > $50 \mathrm{pg} / \mathrm{mL}$ & 0.89 & $0.57-1.39$ & 0.63 & 0.12 & 0.73 & 0.92 & $0.59-1.44$ & 0.72 & 0.04 & 0.84 \\
\hline $\mathrm{eGFR}<60 \mathrm{~mL} / \mathrm{min} / 1.73 \mathrm{~m}^{2}$ & 1.71 & $1.09-2.66$ & $<0.05$ & 0.05 & 0.82 & 1.26 & $0.79-2.01$ & 0.33 & 0.26 & 0.61 \\
\hline $25-\mathrm{OH}-\mathrm{D}<10 \mathrm{ng} / \mathrm{mL}$ & 2.45 & $1.41-4.25$ & $<0.01$ & 0.40 & 0.52 & 2.32 & $1.33-4.02$ & $<0.01$ & 0.26 & 0.61 \\
\hline $\mathrm{CRP}>3.0 \mathrm{mg} / \mathrm{L}$ & 1.47 & $0.91-2.38$ & 0.12 & 0.03 & 0.86 & 1.25 & $0.77-2.03$ & 0.37 & 0.01 & 0.97 \\
\hline Modified Rankin scale ( $\mathrm{mRS}$ ) at admission $\geq 4$ & 3.31 & $1.81-6.03$ & $<0.001$ & 2.69 & 0.10 & 3.20 & $1.76-5.85$ & $<0.001$ & 2.25 & 0.13 \\
\hline $\mathrm{mRS}$ at discharge $=5$ & 7.64 & $4.87-12.01$ & $<0.001$ & 8.55 & $<0.01$ & 6.47 & $3.99-10.49$ & $<0.001$ & 6.81 & $<0.01$ \\
\hline $\mathrm{mRS}$ at discharge $=5$ with time & 1.05 & $1.01-1.09$ & $<0.05$ & - & - & 1.04 & $1.01-1.09$ & $<0.05$ & - & - \\
\hline 25-OH-D 20-29.9 ng/mL & 2.37 & $1.15-4.88$ & $<0.05$ & & & 2.29 & $1.11-4.71$ & $<0.05$ & & \\
\hline 25-OH-D 10-19.9 ng/mL & 5.38 & $2.32-12.47$ & $<0.001$ & 2.41 & 0.49 & 5.01 & $2.15-11.67$ & $<0.001$ & 1.79 & 0.62 \\
\hline $25-\mathrm{OH}-\mathrm{D}<10 \mathrm{ng} / \mathrm{mL}$ & 11.99 & $5.23-27.51$ & $<0.001$ & & & 9.11 & $3.92-21.19$ & $<0.001$ & & \\
\hline NIHSS-I at admission (pts) & 1.10 & $1.06-1.14$ & $<0.001$ & 0.33 & 0.56 & 1.09 & $1.04-1.13$ & $<0.001$ & 0.19 & 0.67 \\
\hline
\end{tabular}

TABLE 4: Results of crude and age-adjusted multivariable stepwise backward Cox proportional hazard regression for death and composite end point.

\begin{tabular}{|c|c|c|c|c|c|c|}
\hline & \multicolumn{3}{|c|}{ Crude analysis } & \multicolumn{3}{|c|}{ Age-adjusted analysis } \\
\hline & $\mathrm{HR}$ & $95 \% \mathrm{CI}$ & $p$ & $\mathrm{HR}$ & $95 \%$ CI & $p$ \\
\hline \multicolumn{7}{|l|}{ Death } \\
\hline Age $>72$ years & 1.80 & $1.08-2.98$ & $<0.05$ & - & - & - \\
\hline $25-\mathrm{OH}-\mathrm{D}<10 \mathrm{ng} / \mathrm{mL}$ & 2.14 & $1.23-3.72$ & $<0.01$ & 2.17 & $1.24-3.77$ & $<0.01$ \\
\hline Modified Rankin scale ( $\mathrm{mRS}$ ) at admission $\geq 4$ & 2.23 & $1.22-4.10$ & $<0.05$ & 2.23 & $1.21-4.11$ & $<0.05$ \\
\hline $\mathrm{mRS}$ at discharge $=5$ & 5.79 & $3.61-9.31$ & $<0.001$ & 5.76 & $3.57-9.29$ & $<0.001$ \\
\hline \multicolumn{7}{|l|}{ Composite end point } \\
\hline Age $>72$ years & 1.70 & $1.09-2.68$ & $<0.05$ & - & - & - \\
\hline $25-\mathrm{OH}-\mathrm{D}<10 \mathrm{ng} / \mathrm{mL}$ & 2.05 & $1.21-3.47$ & $<0.01$ & 2.07 & $1.22-3.50$ & $<0.01$ \\
\hline $\mathrm{mRS}$ at admission $\geq 4$ & 1.95 & $1.05-3.64$ & $<0.05$ & 1.94 & $1.04-3.63$ & $<0.05$ \\
\hline $\mathrm{mRS}$ at discharge $=5$ & 5.08 & $3.22-8.03$ & $<0.001$ & 5.05 & $3.18-8.01$ & $<0.001$ \\
\hline
\end{tabular}

of IS in the Nurses' Health Study comparing women in the lowest versus highest tertiles of $\mathrm{VD}$, with the largest $(\mathrm{OR}=2.47)$ for lacunar infarction. The effects of VD deficiency on the occurrence of hypertension and diabetes may explain this strong association because these diseases are considered major risk factors for lacunar stroke [16]. Although recent meta-analysis suggests that both of these clinical conditions are equally associated with all types of IS [17], we demonstrated similar VD, iPTH, and iFGF23 levels regardless of the type of IS. However, the cardiovascular risk factor profile varies according to the different IS subtypes. Atrial fibrillation and ischemic heart disease are more frequent in patients with cardioembolic infarction, hypertension and diabetes in patients with lacunar stroke, and peripheral artery disease, hypertension, diabetes, and chronic obstructive pulmonary disease in patients with atherothrombotic infarction [18].

Notwithstanding, Zhang et al. have recently shown that $\mathrm{VD}$ deficiency is an independent prognostic factor of functional outcome in IS patients only without hypertension. 
TABLE 5: Results of crude and age-adjusted univariable Cox proportional hazard regression for composite end point.

\begin{tabular}{|c|c|c|c|c|c|c|c|c|c|c|}
\hline & \multicolumn{5}{|c|}{ Cox proportional hazard-crude analysis } & \multicolumn{5}{|c|}{ Cox proportional hazard-age adjusted } \\
\hline & HR & $95 \%$ CI & $p$ & $\chi^{2}$ & $p$ & HR & $95 \% \mathrm{CI}$ & $p$ & $\chi^{2}$ & $p$ \\
\hline Age $>72$ years & 2.40 & $1.52-3.77$ & $<0.001$ & 1.45 & 0.23 & - & - & - & - & - \\
\hline Female vs. male & 1.41 & $0.91-2.18$ & 0.12 & 0.38 & 0.54 & 1.09 & $0.69-1.74$ & 0.69 & 0.14 & 0.70 \\
\hline Diabetes & 1.66 & $1.08-2.56$ & $<0.05$ & 1.98 & 0.16 & 1.48 & $0.96-2.28$ & 0.07 & 1.42 & 0.23 \\
\hline Hypertension & 0.86 & $0.48-1.52$ & 0.61 & 1.23 & 0.27 & 0.71 & $0.40-1.28$ & 0.26 & 0.99 & 0.32 \\
\hline Atrial fibrillation & 1.47 & $0.94-2.28$ & 0.09 & 0.05 & 0.82 & 1.10 & $0.69-1.75$ & 0.67 & 0.15 & 0.69 \\
\hline Ischemic heart disease & 1.56 & $1.01-2.44$ & $<0.05$ & 0.01 & 0.91 & 1.37 & $0.87-2.16$ & 0.17 & 0.01 & 0.92 \\
\hline Valvular diseases & 1.72 & $0.93-3.17$ & 0.08 & 0.22 & 0.64 & 1.49 & $0.80-2.76$ & 0.21 & 0.34 & 0.56 \\
\hline Previous stroke & 1.02 & $0.60-1.71$ & 0.95 & 1.28 & 0.26 & 0.91 & $0.54-1.53$ & 0.72 & 1.27 & 0.26 \\
\hline Hypercholesterolemia & 0.81 & $0.49-1.33$ & 0.40 & 0.09 & 0.76 & 0.85 & $0.52-1.39$ & 0.52 & 0.11 & 0.74 \\
\hline $\mathrm{iPTH} \geq 65 \mathrm{pg} / \mathrm{mL}$ & 1.56 & $0.97-2.52$ & 0.07 & 0.27 & 0.60 & 1.37 & $0.84-2.22$ & 0.20 & 0.63 & 0.43 \\
\hline iFGF23 > $50 \mathrm{pg} / \mathrm{mL}$ & 0.86 & $0.56-1.31$ & 0.48 & 0.02 & 0.89 & 0.88 & $0.57-1.35$ & 0.57 & 0.01 & 0.97 \\
\hline $\mathrm{eGFR}<60 \mathrm{~mL} / \mathrm{min} / 1.73 \mathrm{~m}^{2}$ & 1.63 & $1.06-2.51$ & $<0.05$ & 0.05 & 0.83 & 1.25 & $0.79-1.96$ & 0.33 & 0.25 & 0.61 \\
\hline $25-\mathrm{OH}-\mathrm{D}<10 \mathrm{ng} / \mathrm{mL}$ & 2.38 & $1.41-4.01$ & $<0.01$ & 0.53 & 0.47 & 2.26 & $1.34-3.81$ & $<0.01$ & 0.37 & 0.54 \\
\hline $\mathrm{CRP}>3.0 \mathrm{mg} / \mathrm{L}$ & 1.47 & $0.92-2.35$ & 0.10 & 0.04 & 0.84 & 1.27 & $0.80-2.04$ & 0.31 & 0.01 & 0.97 \\
\hline Modified Rankin scale (mRS) at admission $\geq 4$ & 3.04 & $1.67-5.52$ & $<0.001$ & 3.14 & 0.07 & 2.96 & $1.63-5.39$ & $<0.001$ & 2.64 & 0.10 \\
\hline $\mathrm{mRS}$ at discharge $=5$ & 6.54 & $4.24-10.09$ & $<0.001$ & 9.26 & $<0.01$ & 5.68 & $3.57-9.03$ & $<0.001$ & 7.91 & $<0.01$ \\
\hline mRS at discharge $=5$ with time & 1.04 & $1.01-1.08$ & $<0.05$ & - & - & 1.04 & $0.99-1.08$ & 0.08 & - & - \\
\hline 25-OH-D 20-29.9 ng/mL & 1.88 & $0.99-3.56$ & 0.05 & & & 1.82 & $0.96-3.46$ & 0.07 & & \\
\hline 25-OH-D 10-19.9 ng/mL & 3.96 & $1.83-8.58$ & $<0.001$ & 2.88 & 0.41 & 3.72 & $1.71-8.10$ & $<0.01$ & 2.24 & 0.52 \\
\hline $25-\mathrm{OH}-\mathrm{D}<10 \mathrm{ng} / \mathrm{mL}$ & 8.87 & $4.14-19.00$ & $<0.001$ & & & 6.96 & $3.20-15.11$ & $<0.001$ & & \\
\hline NIHSS-I at admission (pts) & 1.08 & $1.04-1.13$ & $<0.001$ & 0.43 & 0.51 & 1.07 & $1.03-1.12$ & $<0.01$ & 0.30 & 0.58 \\
\hline
\end{tabular}

The reason why the effect of VD deficiency is restricted to patients without hypertension required further study [19].

Some studies suggest that iPTH and VD are separate risk factors for IS [20]. Certainly, secondary hyperparathyroidism may be related to VD deficiency. However, normocalcemic, VD-sufficient hyperparathyroidism is a frequent and increasing with age finding in the population [21]. We showed that secondary hyperparathyroidism is not a significant predictor of mortality after IS or composite end point (recurrent IS or death). Therefore, the effect of $25-\mathrm{OH}-\mathrm{D}$ on high mortality and poor prognosis is probably due to mechanisms beyond calcium-phosphate disturbances. It has been shown that VD affects vasoconstriction by regulating intracellular calcium in vascular smooth muscle cells. Also, arachidonic acid metabolism in the endothelium [21] is disturbed in multiple ways-including modulation of production of proinflammatory cytokines and inhibition of proliferation of inflammatory cells (mostly monocytes), inhibition of macrophage-derived foam cells and angiogenesis, decreasing activation of the reninangiotensin-aldosterone system, and lowering concentrations of 25-OH-D [21-23]. There were also associations with insulin resistance and low HDL-cholesterol levels [14]. It is unclear which of the above mechanisms is most significant with regard to the effect of VD deficiency on high mortality and poor prognosis.

Recently, serum concentration of fibroblast growth factor 23 (FGF23) has emerged as a novel risk factor for IS. Di Giuseppe et al. [24] reported an increased risk for haemorrhage, but not IS, in the top two FGF23 quartiles. It is further demonstrated by Panwar et al. that FGF23 is not a risk factor for IS [25]. Similarly, our study shows that FGF23 levels do not predict mortality after IS or composite end point (recurrent IS or death).

Although the link between VD deficiency and poor prognosis in IS seems to be undisputed, an unanswered question is whether VD supplementation in deficient subjects may improve the prognosis in IS patients by decreasing the risk of recurrent IS and death from other cardiovascular diseases. Several observational studies have reported a protective effect of VD on short-term functional outcome of IS [7, 26-28]. Lower vitamin $\mathrm{D}$ concentration was also proved to be associated with a higher risk of cardiovascular diseases and mortality in the general population [29, 30]; according to our prognostication in this study, effective treatment of 5 persons with severe VD deficiency after stroke may prevent the occurrence of one death or recurrent IS. These estimations are very enthusiastic in nature but are not based on prospective interventional study.

Currently, the only interventional study was performed by Gupta et al. [31]. In this randomized, controlled, openlabel trial, the authors showed a $74 \%$ reduction in mortality after receiving a single intramuscular injection of 600000 IU cholecalciferol in the arm followed by oral cholecalciferol $60000 \mathrm{IU}$ once a month with $1 \mathrm{~g}$ of elemental calcium daily. 
Before we recommend the use of VD in IS patients to improve IS outcomes and not only calcium and bone metabolism, we first must answer several questions: whether and when to start VD supplementation, how long it should be continued, and what is the optimal level for VD regarding prevention of recurrent IS. This requires large, multicenter, randomized trials covering a wide range of age and race subjects living in various geographical areas.

Our study has some limitations. Despite the lack of sample size calculation, the number of patients, both men and women, was sufficient to analyze the influence of severe VD deficiency on the risk of death but insufficient to assess the effect on the IS recurrence. The reference groups for risk calculation were patients with moderate VD insufficiency and deficiency but included few subjects with sufficient VD levels. Finally, out-of-hospital causes of death were not verified by autopsy. Our findings concerning the prevalence of vitamin $\mathrm{D}$ deficiency should not be generalized and restricted to white Caucasians living in the same area.

\section{Conclusion}

Severe VD deficiency is an emerging, strong negative predictor for survival after IS, independent of age and functional status. VD supplementation in stroke survivals may be considered. There is a need to perform a clinical randomized controlled study to demonstrate whether VD supplementation improves an IS patient's survival.

\section{Abbreviations}

$\begin{array}{ll}\text { IS: } & \text { Ischemic stroke } \\ \text { VD: } & \text { Vitamin D } \\ \text { 25-OH-D: } & \text { 25-Hydroxyvitamin D } \\ \text { iPTH: } & \text { Intact parathyroid hormone } \\ \text { iFGF23: } & \text { Intact fibroblast growth factor 23 } \\ \text { iNOS: } & \text { Inducible nitric oxide synthase } \\ \text { CT: } & \text { Computed tomography } \\ \text { NIHSS: } & \text { National Institute of Health Stroke Scale } \\ \text { mRS: } & \text { Modified Rankin scale } \\ \text { TOAST: } & \text { Trial of Org 10172 in Acute Stroke } \\ & \text { Treatment } \\ \text { ADL: } & \text { Activities of daily living } \\ \text { eGFR: } & \text { Estimated glomerular filtration rate } \\ \text { MDRD: } & \text { Modification of diet in renal disease } \\ \text { pH: } & \text { Proportional hazard } \\ \text { PAR: } & \text { Population attributable risk } \\ \text { NNT: } & \text { Number needed to treat } \\ \text { CEP: } & \text { Composite end point } \\ \text { HDL-cholesterol: } & \text { High-density lipoprotein cholesterol. }\end{array}$

\section{Data Availability}

The datasets analysed during the current study are not publicly available due to the continuation of analyses but are available from the corresponding author on reasonable request.

\section{Ethical Approval}

The study was approved by the Bioethics Committee of the Medical University of Silesia.

\section{Consent}

Consent was provided upon request.

\section{Disclosure}

The study was presented as a poster during the 13th International Congress of the European Union Geriatric Medicine Society (printed version of the abstract available at European Geriatric Medicine 2017: 8S1; S40-S247).

\section{Conflicts of Interest}

The authors declare that they have no competing interests.

\section{Authors' Contributions}

JW was responsible for data acquisition and drafting of the manuscript. MS was responsible for data interpretation and critical revision for important intellectual content. AJO was responsible for data analysis and statistical analysis. $\mathrm{AB}$ was responsible for laboratory analyses. MOG was responsible for critical revision for important intellectual content. JC was responsible for conception and design, obtaining funds, data interpretation, manuscript editing, and final approval. All authors have read and approved the final version of the manuscript.

\section{Acknowledgments}

We are very grateful to Randy Jackson for the English edition of the manuscript. The project was funded by the Medical University of Silesia grant no. KNW-1-072/N/6/0.

\section{References}

[1] WHO 2006, Neurological Disorders: Public Health Challenges, World Health Organization, Geneva, Switzerland, 2006, January 2017, http://www.who.int/mental_health/neurology/ neurodiso/en/.

[2] Y. Béjot, H. Bailly, J. Durier, and M. Giroud, "Epidemiology of stroke in Europe and trends for the 21st century," La Presse Médicale, vol. 45, no. 12, pp. e391-e398, 2016.

[3] H. Ay, E. M. Arsava, G. Andsberg et al., "Pathogenic ischemic stroke phenotypes in the NINDS-stroke genetics network," Stroke, vol. 45, no. 12, pp. 3589-3596, 2014.

[4] M. F. Holick, "Vitamin D deficiency," The New England Journal of Medicine, vol. 357, no. 3, pp. 266-281, 2007.

[5] D. E. Wallis, S. Penckofer, and G. W. Sizemore, "The "sunshine deficit" and cardiovascular disease," Circulation, vol. 118, no. 14, pp. 1476-1485, 2008.

[6] J. H. Lee, J. H. O'Keefe, D. Bell, D. D. Hensrud, and M. F. Holick, "Vitamin D deficiency: an important, common, and easily treatable cardiovascular risk factor?," Journal of the American College of Cardiology, vol. 52, no. 24, pp. 19491956, 2008. 
[7] K. Y. Park, P. W. Chung, Y. B. Kim et al., "Serum vitamin D status as a predictor of prognosis in patients with acute ischemic stroke," Cerebrovascular Diseases, vol. 40, no. 1-2, pp. 73-80, 2015.

[8] E. Giovannucci, Y. Liu, B. W. Hollis, and E. B. Rimm, "25-Hydroxyvitamin D and risk of myocardial infarction in men," Archives of Internal Medicine, vol. 168, no. 11, pp. 1174-1180, 2008.

[9] J. S. Buell and B. Dawson-Hughes, "Vitamin D and neurocognitive dysfunction: preventing "D"ecline?," Molecular Aspects of Medicine, vol. 29, no. 6, pp. 415-422, 2008.

[10] E. C. Jauch, J. L. Saver, H. P. Adams et al., "Guidelines for the early management of patients with acute ischemic stroke: a guideline for healthcare professionals from the American Heart Association/American Stroke Association," Stroke, vol. 44, pp. 870-947, 2013.

[11] H. P. Adams Jr, B. H. Bendixen, L. J. Kappelle et al., "Classification of subtype of acute ischemic stroke: definitions for use in a multicenter clinical trial," Stroke, vol. 24, no. 1, pp. 35-41, 1993.

[12] M. Wyskida, A. Owczarek, A. Szybalska et al., "Socioeconomic determinants of vitamin D deficiency in the older Polish population: results from the PolSenior study," Public Health Nutrition, vol. 21, no. 11, pp. 1995-2003, 2018.

[13] J. C. Gallagher and A. J. Sai, "Vitamin D insufficiency, deficiency, and bone health," The Journal of Clinical Endocrinology \& Metabolism, vol. 95, no. 6, pp. 2630-2633, 2010.

[14] R. C. Strange, K. E. Shipman, and S. Ramachandran, "Metabolic syndrome: a review of the role of vitamin D in mediating susceptibility and outcome," World Journal of Diabetes, vol. 6, no. 7, pp. 896-911, 2015.

[15] Q. Sun, A. Pan, F. B. Hu, J. A. E. Manson, and K. M. Rexrode, "25-Hydroxyvitamin D levels and the risk of stroke: a prospective study and meta-abalysis," Stroke, vol. 43, no. 6, pp. 14701477, 2012.

[16] A. Arboix and J. L. Marti-Vilalta, "Lacunar stroke," Expert Review of Neurotherapeutics, vol. 9, no. 2, pp. 179-196, 2009.

[17] C. A. Jackson, A. Hutchison, M. S. Dennis et al., "Differing risk factor profiles of ischemic stroke subtypes: evidence for a distinct lacunar arteriopathy?," Stroke, vol. 41, no. 4, pp. 624-629, 2010.

[18] A. Arboix, "Cardiovascular risk factors for acute stroke: risk profiles in the different subtypes of ischemic stroke," World Journal of Clinical Cases, vol. 3, no. 5, pp. 418-428, 2015.

[19] B. Zhang, Y. Wang, Y. Zhong, S. Liao, and Z. Lu, "Serum 25-hydroxyvitamin D deficiency predicts poor outcome among acute ischemic stroke patients without hypertension," Neurochemistry International, vol. 118, pp. 91-95, 2018.

[20] G. Kontogeorgos, P. Trimpou, C. M. Laine, G. Oleröd, A. Lindahl, and K. Landin-Wilhelmsen, "Normocalcaemic, vitamin $\mathrm{D}$-sufficient hyperparathyroidism - high prevalence and low morbidity in the general population: a long-term follow-up study, the WHO MONICA project, Gothenburg, Sweden," Clinical Endocrinology, vol. 83, no. 2, pp. 277284, 2015.

[21] L. S. F. Carvalho and A. C. Sposito, "Vitamin D for the prevention of cardiovascular disease:are we ready for that?", Atherosclerosis, vol. 241, no. 2, pp. 729-740, 2015.

[22] V. Nsengiyumva, M. E. Fernando, J. V. Moxon et al., “The association of circulating 25-hydroxyvitamin D concentration with peripheral arterial disease: a meta-analysis of observational studies," Atherosclerosis, vol. 243, no. 2, pp. 645-651, 2015.

[23] M. Takeda, T. Yamashita, N. Sasaki et al., "Oral administration of an active form of vitamin D3 (calcitriol) decreases atherosclerosis in mice by inducing regulatory $\mathrm{T}$ cells and immature dendritic cells with tolerogenic functions," Arteriosclerosis, Thrombosis, and Vascular Biology, vol. 30, no. 12, pp. 24952503, 2010.

[24] R. di Giuseppe, T. Kühn, F. Hirche et al., "Plasma fibroblast growth factor 23 and risk of cardiovascular disease: results from the EPIC-Germany case-cohort study," European Journal of Epidemiology, vol. 30, no. 2, pp. 131-141, 2015.

[25] B. Panwar, N. S. Jenny, V. J. Howard et al., "Fibroblast growth factor 23 and risk of incident stroke in community-living adults," Stroke, vol. 46, no. 2, pp. 322-328, 2015.

[26] Y. Wang, H. Ji, Y. Tong, and Z. B. Zhang, "Prognostic value of serum 25-hydroxyvitamin D in patients with stroke," Neurochemical Research, vol. 39, no. 7, pp. 1332-1337, 2014.

[27] W. J. Tu, S. J. Zhao, D. J. Xu, and H. Chen, "Serum 25hydroxyvitamin D predicts the short-term outcomes of Chinese patients with acute ischaemic stroke," Clinical Science, vol. 126, no. 5, pp. 339-346, 2014.

[28] B. Daubail, A. Jacquin, J. C. Guilland et al., "Serum 25hydroxyvitamin D predicts severity and prognosis in stroke patients," European Journal of Neurology, vol. 20, no. 1, pp. 57-61, 2013.

[29] K. T. Khaw, R. Luben, and N. Wareham, "Serum 25hydroxyvitamin D, mortality, and incident cardiovascular disease, respiratory disease, cancers, and fractures: a 13-y prospective population study," The American Journal of Clinical Nutrition, vol. 100, no. 5, pp. 1361-1370, 2014.

[30] A. Kilkkinen, P. Knekt, A. Aro et al., "Vitamin D status and the risk of cardiovascular disease death," American Journal of Epidemiology, vol. 170, no. 8, pp. 1032-1039, 2009.

[31] A. Gupta, S. Prabhakar, M. Modi et al., "Effect of vitamin D and calcium supplementation on ischaemic stroke outcome: a randomised controlled open-label trial," International Journal of Clinical Practice, vol. 70, no. 9, pp. 764-770, 2016. 


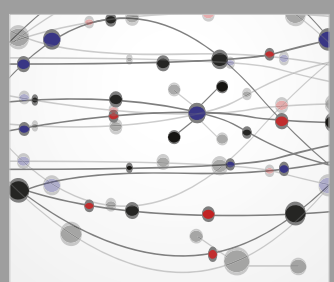

The Scientific World Journal
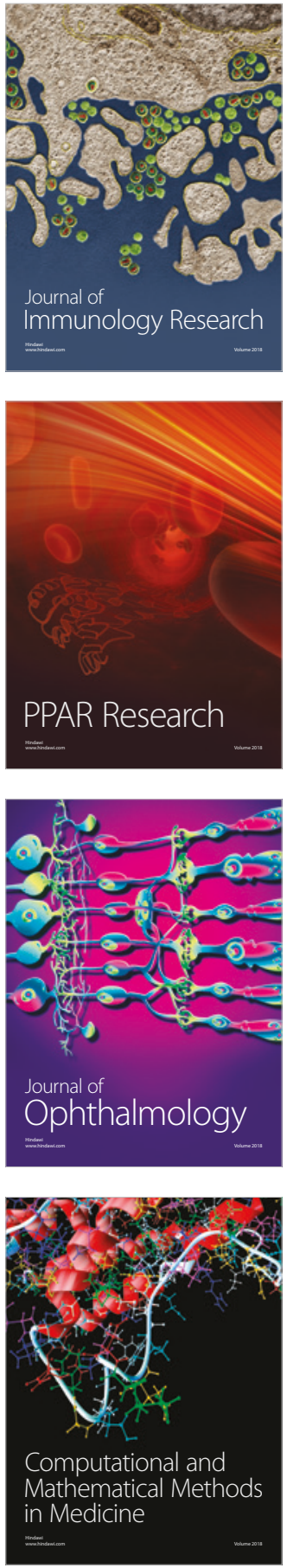

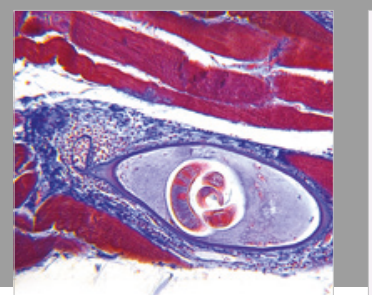

Gastroenterology Research and Practice

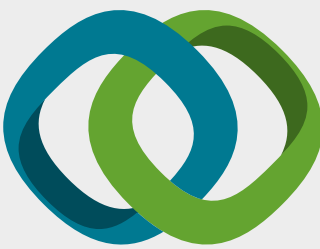

\section{Hindawi}

Submit your manuscripts at

www.hindawi.com
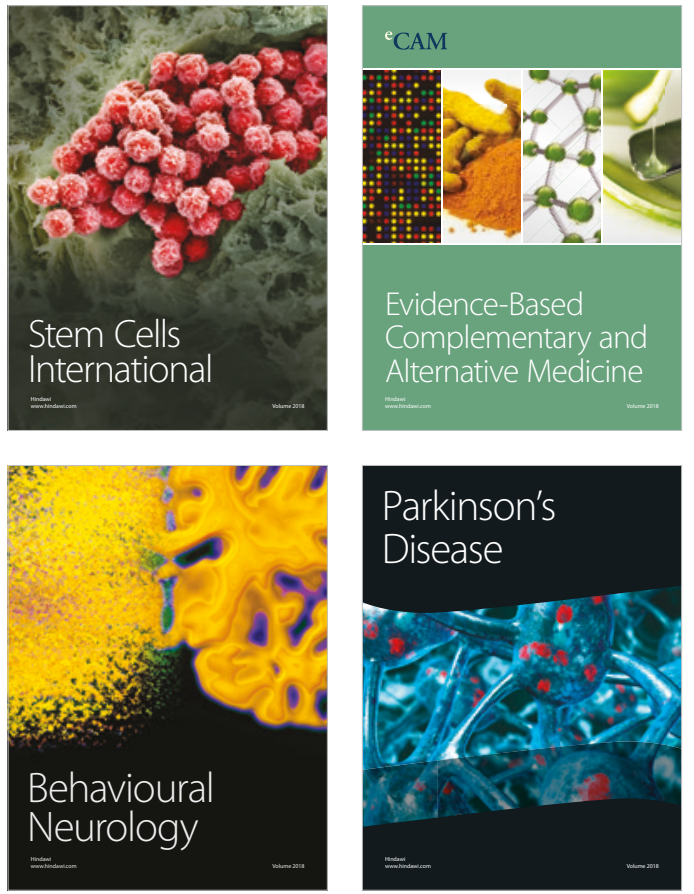

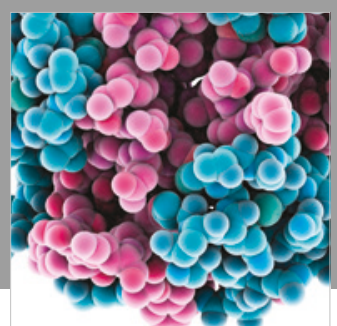

ournal of

Diabetes Research

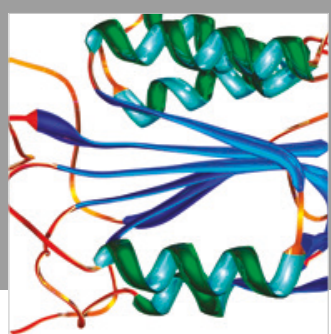

Disease Markers
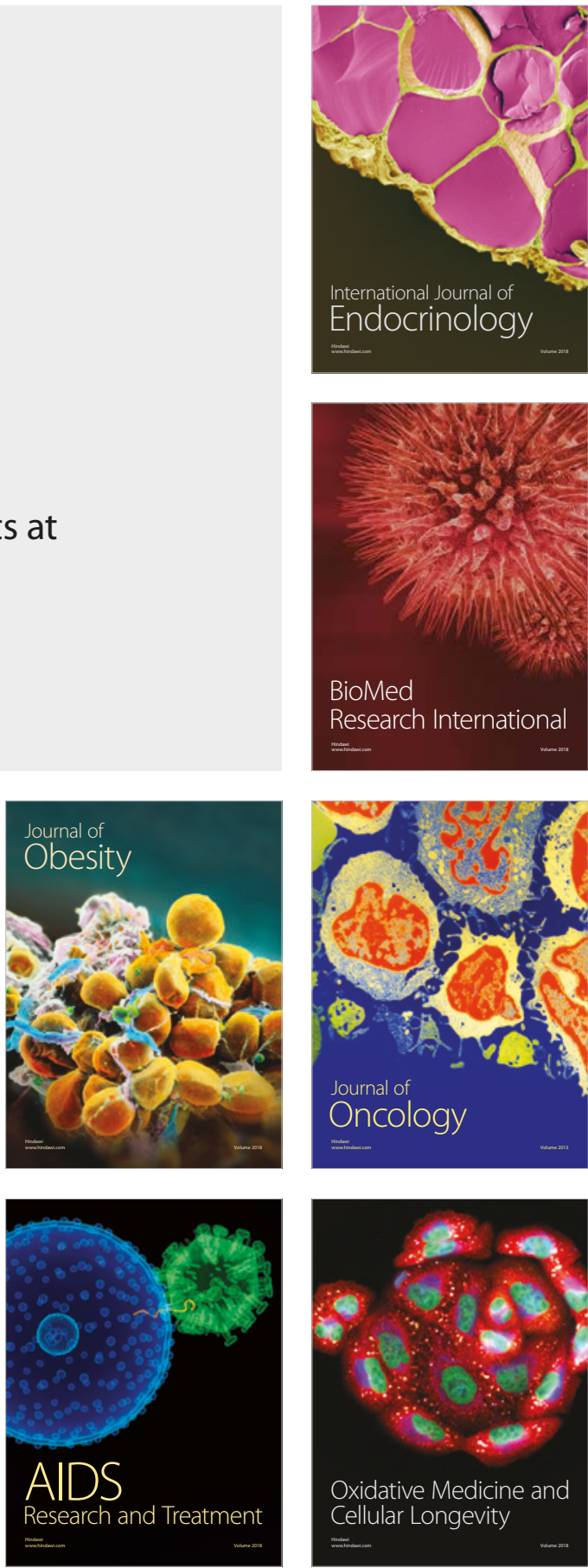